\title{
An Adaptation Algorithm for an Intelligent Natural Language Tutoring System
}

\begin{abstract}
The focus of computerised learning has shifted from content delivery towards personalised online learning with Intelligent Tutoring Systems (ITS). Oscar Conversational ITS (CITS) is a sophisticated ITS that uses a natural language interface to enable learners to construct their own knowledge through discussion. Oscar CITS aims to mimic a human tutor by dynamically detecting and adapting to an individual's learning styles whilst directing the conversational tutorial. Oscar CITS is currently live and being successfully used to support learning by university students. The major contribution of this paper is the development of the novel Oscar CITS adaptation algorithm and its application to the Felder-Silverman learning styles model. The generic Oscar CITS adaptation algorithm uniquely combines the strength of an individual's learning style preference with the available adaptive tutoring material for each tutorial question to decide the best fitting adaptation. A case study is described, where Oscar CITS is implemented to deliver an adaptive SQL tutorial. Two experiments are reported which empirically test the Oscar CITS adaptation algorithm with students in a real teaching/learning environment. The results show that learners experiencing a conversational tutorial personalised to their learning styles performed significantly better during the tutorial than those with an unmatched tutorial.
\end{abstract}

\section{Keywords:}

Human-computer interface

Intelligent tutoring systems

Interactive learning environments

Teaching/learning strategies

\section{Introduction}

ITS build on the conventional computerised learning systems' 'one-size-fits-all' approach by capturing and modelling individual characteristics used to personalise the instruction (Stankov, Rosić, Žitko \& Grubišić 2008). This involves presenting learning material in a style and order to suit the learner (e.g. by presenting learning material matched to poorly understood topics), and also proactively helping learners, e.g. by giving intelligent feedback on incomplete or erroneous solutions and guidance to assist learners in constructing solutions to problems. Most ITS are student-led with menus or hyperlinks to topics, sometimes designed specifically to capture individual traits (Cha, Kim, Park, Yoon, Jung \& Lee 2006). The increasing complexity of computer systems and their interfaces has stimulated a move towards creating intuitive, human-centred interfaces, such as natural language dialogue via conversational agents (CAs) (O’Shea, Bandar \& Crockett 2011). Learning is inherently a social process (Wang \& Wu 2008; Jones \& Issroff 2005) and during face-to-face tutorials human tutors automatically pick up behavioural cues from students about their understanding and learning preferences, and adapt their teaching style to aid learning. A few ITS attempt to mimic a human tutor by including CA interfaces that promote the construction of knowledge through discussion (Dzikovska, Bental, Moore, Steinhauser, Campbell, Farrow et al. 2010; Graesser, Chipman, Haynes \& Olney 2005; Rahati \& Kabanza 2010). Such ITS (known as Conversational ITS (CITS)) have now been extended to detect and react to learners' emotions (Lehman, D’Mello \& Graesser 2012) and learning styles (Latham, Crockett, McLean \& Edmonds 2012a). Learning styles model the way that groups of people prefer to receive and process information, e.g. by viewing diagrams rather than textual descriptions (Felder \& Silverman 1988; Honey \& Mumford 1992). Research in computer-based education systems has shown that adapting the style of tutoring to match student learning styles can enhance learning (Akbulut \& Cardak 2012; Tsianos, Lekkas, Germanakos, Mourlas \& Samaras 2008).

Oscar CITS is an innovative Conversational Intelligent Tutoring System that can imitate a human tutor by directing a tutoring conversation and dynamically detecting and adapting to student learning styles during the conversation (Crockett, Latham, McLean, Bandar \& O'Shea 2011; Latham et al. 2012a; Latham, Crockett, McLean \& Edmonds 2012b). Oscar's tutor-led, conversational approach enables learners to actively discuss problems and collaboratively arrive at solutions, supporting the constructivist style of learning adopted by human tutors. Oscar CITS aims to improve each person's learning experience by using artificial intelligence techniques to adapt its tutoring style to match their existing knowledge and preferred learning style. During the tutorial conversation, Oscar CITS uses prompts and hints to encourage learners to solve problems by themselves and gives instant individual feedback on errors and omissions. Oscar CITS is a live system being successfully used to support learning by students in the School of Computing, Mathematics and Digital Technology at Manchester Metropolitan University.

$<$ FIG. 1 HERE $>$

Oscar CITS is a large and complex system that was designed with a modular architecture to separate the 
domain and learning styles knowledge from the functionality (see Fig. 1). Oscar CITS' general, modular design facilitates its independence from the tutorial subject domain and learning styles model. A full description of the Oscar CITS architecture and components is given in (Latham et al. 2012b) and summarised here. A central controller module manages the learner interaction and the communication between components. The GUI manages the webpage interaction with the user, displaying responses, images and movies and reacting to user events. The student model manages all information about individual learners, including knowledge, topics visited, test scores and learning styles. The CA accepts a natural language utterance and uses student, learning styles and tutorial knowledge to generate a natural language response. The CA uses a database of scripts containing pattern-based rules that match a stimulus input to a response. Designing the rules is a demanding task: the case study that will be described in Section 4 required 95 scripts containing about 1000 rules, each containing multiple patterns. The tutorial knowledge base manages all course information, such as topic breakdowns, teaching materials and tests and is related but not linked to the CA scripts. The Oscar CITS can be implemented for new subjects by plugging in a different tutorial knowledge base and CA scripts. The learning styles predictor agent uses knowledge of a learning styles model to predict an individual's preferred learning styles from their dialogue and behaviour. The learning styles adapter agent uses knowledge of learning styles, tutorial knowledge and learner preferences to decide the best adaptation to apply for each question. Different learning styles models can be applied to Oscar CITS by replacing the learning styles predictor and adapter agents.

The complex task of the design, development and experimental analysis of Oscar CITS was managed by separating the functionality of learning styles prediction and adaptation. The Oscar CITS predictive subsystem (incorporating the Learning Styles Predictor Agent) was able to implicitly predict an individual's preferred learning styles by capturing aspects of their behaviour and dialogue during the conversation (Crockett et al. 2011; Latham et al. 2012a). The formal methodology for developing the Oscar CITS predictive subsystem to deliver a real time conversational tutorial for SQL is detailed in (Latham et al. 2012a). Several experiments involving real undergraduate and postgraduate students were conducted in a real educational setting to investigate the Oscar CITS predictive subsystem's ability to automatically model learning styles (Latham et al. 2012a). The results show that the Oscar CITS predictive subsystem successfully predicted all eight learning styles in the Felder-Silverman (FS) learning styles model (Felder \& Silverman 1988) with accuracies of 61$100 \%$.

This paper expands on the development methodology for the Oscar CITS adaptive subsystem reported in (Latham et al. 2012b) to describe the design and implementation of the novel Oscar CITS adaptation algorithm. The main contributions of this paper are:

- The design of Oscar CITS innovative approach to adaptation, which selects the best fitting adaptation for each tutorial question, resulting in tutorials containing a variety of teaching styles to promote student engagement. Oscar's generic approach can be applied to individual social factors such as learning style or emotion to provide a personalised conversational tutorial.

- The development of the generic Oscar CITS adaptation algorithm, which uniquely takes into account the availability of adaptive tutoring material for each tutoring question as well as the learner's preferences. The Oscar CITS adaptation algorithm allows several different individual factors to be combined to determine the best fitting adaptation (e.g. several different aspects of learning style).

- The application of the general Oscar CITS adaptation algorithm to the Felder-Silverman learning styles model.

- A case study describing the implementation of Oscar CITS for an SQL tutoring knowledge base, delivering a conversational tutorial which adapts to individual users' learning styles (using the FS model).

- The description of two experiments to empirically test the success of the Oscar CITS adaptation algorithm in a real life teaching/learning environment.

This paper is organised as follows: Section 2 describes the three research areas brought together by this research, namely intelligent tutoring systems, conversational agents and learning styles and reviews relevant related work. Section 3 describes Oscar CITS and the current state of its implementation, a discussion of the Oscar CITS adaptation approach and its generic adaptation algorithm. Section 4 presents a case study of Oscar CITS that implements the adaptation algorithm to deliver a personalised conversational tutorial for the domain of SQL adapting to individual learning style preference. Section 5 describes the empirical evaluation of the adaptation algorithm in a real teaching/learning environment and discusses the results. Section 6 discusses how the Oscar CITS adaptation algorithm may be generalised to incorporate other individual traits and for different domains. Section 7 concludes the paper with a summary and discussion of the study's contributions and limitations.

\section{Background and related work}




\subsection{Conversational agents}

Conversational agents (CAs) enable people to interact with computer systems in real time using natural language dialogues. In expert systems, such as tutoring (Graesser et al. 2005), CA interfaces can engage users in discussion by replicating human communication (e.g. classroom learning), helping users to build motivation and confidence by drawing on their own experience. CA research is an active and complex field with the key challenge of adequately understanding a user input to produce an appropriate response (O'Shea et al. 2011). There are two main approaches to understanding user input:

- Semantic-based CAs endeavour to understand the meaning of an utterance by analysing the constructs and meaning of natural language (Lee, Isa, Choo \& Chue 2012) or by scoring the semantic similarity of phrases (Li, Bandar, McLean \& O’Shea 2004).

- Pattern matching CAs (Wallace 2009; Convagent Ltd 2005) use an algorithm to match key words and phrases within an utterance to a knowledge base of pattern-based stimulus-response pairs rather than endeavouring to understand the input. Although limited to existing stimulus patterns, the pattern matching approach does not require grammatically correct or complete input. However, developing a set of stimulusresponse pairs (known as a script) is a skilled and labour intensive task.

Most text-based CAs adopt the pattern matching approach as it is currently the one that works best for extended dialogues (O'Shea et al. 2011). The pattern matching approach involves the development of conversation scripts that match key words and phrases with appropriate responses. CA scripts may be grouped into topics and may be linked together in a tree or graph structure (Sammut 2001), sometimes over various levels (e.g. a script filter to capture abusive language). Each CA script usually contains a large number of patterns, resulting in many hundreds of stimulus-response pairs in the CA's knowledge base. The organisation of CA scripts and the efficiency of the matching algorithm have a direct impact on the real-time use of CAs as interfaces.

The Oscar CITS described in this paper adopts the InfoChat (Convagent Ltd 2005) pattern-matching textbased CA to deliver the personalised tutorial.

\subsection{Intelligent Tutoring Systems}

Intelligent Tutoring Systems (ITS) are adaptive educational systems that employ artificial intelligence techniques to provide individualised instruction (Graesser et al. 2005). ITS build a model of the goals, preferences and knowledge of the student, and use this to adapt the teaching to the individual and to provide intelligent assistance. There are three main methods of incorporating intelligence in ITS (Brusilovsky \& Peylo 2003):

- Curriculum sequencing systems adapt to individuals by presenting students with learning material in a sequence and style which best suits their needs. Curriculum sequencing is the technique most widely used by adaptive educational systems (Brusilovsky \& Peylo 2003). Originally, personalisation based on existing knowledge aimed to improve the learning experience by tutoring on unknown or weak topics (Stankov, Rosić, Žitko \& Grubišić 2008). Recently curriculum sequencing has been broadened to consider other individual traits that might affect learning, such as the learner's emotions (Lehman et al. 2012; Mao \& Li 2010), personality type (Leontidis, Halatsis \& Grigoriadou 2011) and learning style (Popescu 2010).

- Intelligent solution analysis enables ITS to give detailed feedback on incomplete or erroneous solutions, helping students learn from their mistakes (Pahl \& Kenny 2009).

- Problem solving support embraces the constructivist approach to teaching used by human tutors to intelligently prompt learners to solve problems themselves, constructing their own knowledge and thus encouraging a deeper understanding of a topic (Redondo, Bravo, Ortega \& Verdejo 2007).

ITS commonly adapt by presenting learning material matched to student preferences with the aim of making learning easier and more effective, adding a human-like social intelligence to ITS. Although the nature of learning styles has been questioned by some authors (Coffield, Moseley, Hall \& Ecclestone 2004), extending ITS to adapt tutoring based on individuals' preferred learning styles has been shown to enhance students' learning experiences (Kelly \& Tangney 2006; Sangineto, Capuano, Gaeta \& Micarelli 2007; Triantafillou, Pomportsis, Demetriadis \& Georgiadou 2004).

ITS are usually student-directed, with a list of hyperlinks that are reordered or ranked to recommend a particular sequence to learners (Klasnja-Milicevic, Vesin, Ivanovic \& Budimac 2011). However, rather than actively teaching such designs assist in self-learning, and require students to be self-motivated. Although less common, conversational interfaces allow a more natural, tutor-led learning experience that promotes collaborative problem solving and the construction of knowledge used by human tutors (Chi, Siler, Jeong, Yamauchi \& Hausmann 2001). Conversational ITS (CITS) can act as personal tutors that are available at any time and place, and which provide instant answers to questions, feedback, and support. Moreno, Mayer, Spires \& Lester (2001) suggest that as learning is an "inherently social process", using CITS "learners interpret their 
relation with the computer as a social one involving reciprocal communication". To mimic a human tutor, "it seems necessary for future generations of ITSs to incorporate natural language capabilities." (Chi et al. 2001).

\subsection{The Felder-Silverman Learning Styles Model}

The Felder-Silverman (FS) learning styles model (Felder \& Silverman 1988) describes the learning styles of engineering students over four dimensions relating to steps in the process of receiving and understanding information (see Fig. 2).

$<$ FIG. 2 HERE $>$

Each learning style dimension has two divergent learning styles, giving 8 different learning styles with 16 (24) combinations, e.g. intuitive/verbal/active/sequential. The FS model is measured using the Index of Learning Styles (ILS) instrument (Felder \& Soloman 2008), which is a questionnaire that places learners along each dimension axis according to the strength of their preference for a particular learning style. Low scores indicate a weak preference for a particular style, called neutral preferences, and such neutral learners are placed at the centre of the axis.

The FS model has been adopted by many of the Intelligent Tutoring Systems (ITS) that adapt to student learning styles (Akbulut \& Cardak 2012; Garcia, Amandi, Schiaffino \& Campo 2007) as it offers the following benefits to ITS:

- it includes detailed descriptions of learning behaviour and associated teaching styles;

- it has a small number of dimensions that are distinct and independent;

- $\quad$ it describes engineering students who often make up experimental groups.

The FS model was incorporated into the Oscar CITS for the case study presented in this paper as the initial experimental groups were engineering students. However, the Oscar CITS structure is independent of a particular learning styles model.

\section{Oscar CITS}

Oscar CITS is a sophisticated, novel CITS that can implicitly predict and adapt to an individual's preferred learning style whilst holding a tutoring conversation. The pedagogical aim is to enhance the learning experience by providing the learner with tutoring material suited to their learning styles. Oscar CITS aims to mimic a human tutor by leading a tutoring dialogue and employing intelligent techniques to assist learners in constructing a deeper understanding of the topic. Oscar's natural language interface is familiar and intuitive to learners, allowing the exploration of problems and helping to build confidence and motivation. Like a human tutor, Oscar CITS directs each tutorial, personalising the curriculum depending on an individual's knowledge, responses and learning styles. Oscar CITS is available online, allowing learners to study when and where they choose at a fixed cost.

\subsection{Oscar CITS Approach for Selecting the Best Adaptation}

The Oscar CITS is the only CITS that automatically adapts a tutoring conversation to an individual's learning styles. Learning style models normally describe several aspects (or dimensions) of learner preference relating to stages of the learning process (Felder \& Silverman 1988; Honey \& Mumford 1992). Although no other CITS adapts to learning styles, there are a number of approaches used by less sophisticated ITS to adapt to an individual's preferred learning styles. The literature does not provide details of adaptation algorithms, but the simplest and most common adaptation strategy is to adapt to a single aspect or subset of learning style (Sanders \& Bergasa-Suso 2010; Kelly \& Tangney 2006). The disadvantage of selecting only one learning style is that this does not completely describe the learner's preferences, e.g. in the FS model there are four learning style dimensions. With this approach it is difficult to decide which aspects of learning style to model, and such ITS can only partially address an individual's learning preferences. An adaptation algorithm that selects a variety of learning resources based on several aspects of a person's learning style may provide a richer learning experience, but there are also difficulties when adapting to more than one aspect of learning style:

- The requirement for multiple versions of learning resources to suit different learning styles is a barrier to the development of an ITS. For example, in the FS model there are 16 separate learning styles.

- When developing an adaptive CITS tutorial, it may not be appropriate to incorporate all categories of teaching material into every tutorial question and still present a coherent learning experience, so some learning styles may not be addressed.

- To overcome these problems, one strategy is to adapt to the strongest learning style (Kelly \& Tangney 2006) thus reducing the number of adaptive resources required, e.g. the FS model has four dichotomous dimensions, so eight versions of adaptive resource would be needed. However, adaptation is still only partial, and by adapting to the single most preferred learning style, learners are only presented with one type of learning resource. With this method, the development barrier of producing teaching material is reduced (but not eliminated) and additional strategies are required to deal with conflict when an individual's 
preference for several learning styles is equally strong. Some ITS (Kelly \& Tangney 2006; Carver, Howard \& Lane 1999) have addressed conflict by relying on learners' initiative to select different types of learning resource from a list of links.

Therefore it was concluded that the Oscar CITS adaptation strategy should consider both the strength of the individual's learning style and the availability of adaptive learning material for each individual tutorial question. This would mean that each learner could be presented with the most suitable tutoring material by considering all of their preferred learning styles and also the existing adaptations for every question in the tutorial. The implication of such an adaptation mechanism for learners is that they are presented with the best fitting tutoring material whilst still encountering a coherent learning experience. Learners may also experience more than one learning style adaptation during a tutorial, and such a variation in style can potentially help to improve their interest and motivation (Akbulut \& Cardak 2012; Felder \& Brent 2005). The implication of the selected adaptation strategy for developers is that there is no need to develop multiple versions of teaching materials all at once as tutorials that contain adaptations for only some learning styles can still be successfully used whilst the learning material is built up over time. As well as addressing an important development barrier by allowing partially complete adaptations to be used, such an approach allows tutorials to be tested and changed at an early stage before too much time has been committed to the development.

The Oscar CITS adaptation approach is considerably more sophisticated than adapting to a single aspect of a person's learning style, requiring an algorithm for conflict resolution when more than one learning style dimension wins. However, the Oscar CITS adaptation approach aims to offer a more rounded and complete learning experience whilst still presenting a coherent tutorial.

\subsection{Engineering the Adaptive Tutorial Knowledge}

To implement the strategy of deciding on the best available adaptation for each tutorial question, it was first necessary to develop a method of capturing and storing the knowledge of available adaptations. The FS model was adopted as exemplar learning styles model as it has eight distinct learning styles and contains descriptions of associated preferred teaching styles. The first step was to compile a list of all descriptions of teaching styles given in the FS model. Next, a team of three experts was asked to consider the list of teaching styles and develop categories of teaching material. The team comprised three computing lecturers, each with a teaching qualification, each having a minimum of 18 years of teaching experience, with one lecturer being an Information Systems curriculum leader and author of an international undergraduate textbook on Databases. The lecturers were asked to independently group the extracted teaching styles knowledge into distinct categories of teaching material which would, in their expert opinion, support the teaching styles. The team then met as a committee to discuss, compare and agree a set of teaching material categories which would address all learning styles (see Table 1). Each teaching material category was then mapped to the associated learning styles, as defined in the FS model.

$<$ TABLE 1 HERE $>$

A simple adaptation scoring mechanism was designed to clearly represent the possible adaptations for any particular tutorial question, as follows:

For each tutorial question:

1) Assign each teaching material category (defined in Table 1) a score by counting the number of occurrences of learning objects of that style created for the tutorial question (see example below). Note that each category may occur several times over the course of the conversation for a question, e.g. if several different practical examples are given.

2) Using the learning style mappings in Table 1, for each learning style derive a score by summing the scores assigned to all associated teaching material categories. A score of 0 indicates that no adaptations are available for that learning style.

Using this mechanism, each tutorial question has an adaptation score for every learning style. These scores indicate which learning styles have available adaptations (i.e. a score greater than 0 ) and also the strength of (i.e. number of) adaptation available for each learning style. For example, if a question includes a textual explanation of a theory (category 2 ), three practical examples (category 4 ) and a movie (category 6):

- after step 1 above, the teaching material category scores are: category $2=1$; category $4=3$; category $6=1$; all other categories $(1,3,5,7,8,9)=0$

- after step 2 above, by summing the scores for each learning style mapped to these categories, the adaptation scores derived for the tutorial question are: Sensory 3; Intuitive 2; Visual 1; Verbal 1; Active 3; Reflective 1; Sequential 4; Global 0.

\subsection{Oscar CITS Adaptation Algorithm}

A novel adaptation algorithm was developed that selects the best fitting adaptation per question independently 
of the tutorial domain and the learning styles model adopted. While developing the adaptation algorithm, the FS model was used as an example learning styles model with four learning style dimensions. The assessment of FS learning styles results in a learning style preference being assigned for each dimension, however the FS model states that the group of students who have no strong preference for any learning style should be shown learning material containing a mix of styles. Taking this into consideration, an additional Neutral learning style category was created to group learners with a low preference on all FS dimensions. Such neutral learners will be presented with a Neutral adaptation that includes a mixture of teaching material styles.

A logical representation of the generic Oscar CITS adaptation algorithm for selecting the best adaptation per question is given in Appendix A. For each learning style class in the model, the algorithm calculates a learner question score by combining the strength of the learning style preference with the question adaptation score (explained in Section 3.2). The learning style class with the highest learner question score wins. In the event of conflict (i.e. where there is no clear winner), additional conflict resolution rules are followed:

1. The learning style with the strongest preference is selected.

2. If there is no clear winner, the learning style with the most adaptations is selected.

3. If there is still no clear winner, a winner is selected randomly.

Appendix B demonstrates the algorithm using examples, showing the adaptations selected by the algorithm (applied to the FS model) and the resolution of conflict.

The next section will present a case study describing an implementation of Oscar CITS for the SQL domain to empirically validate the Oscar CITS adaptation algorithm.

\section{Case Study: Creation and Delivery of a Personalised Conversational Tutorial for SQL using Oscar CITS}

This section describes the implementation of Oscar CITS and how it was applied to the SQL domain to empirically test the adaptation algorithm. SQL is a standard database language taught as part of many computer science degree courses. Oscar CITS was implemented to deliver a real time conversational SQL tutorial and integrated into several undergraduate and postgraduate computing modules at Manchester Metropolitan University (MMU). Oscar CITS is currently being used to support undergraduate and postgraduate students in a real teaching/learning environment. The implementation of Oscar CITS and its architecture was reported in (Latham et al. 2012b), so this section will focus on the implementation of the adaptation algorithm. The development of the Oscar CITS adaptive subsystem for an SQL tutorial followed a formal three-phase methodology (Table 2) detailed in (Latham 2011) and summarised below:

\section{$<$ TABLE 2 HERE>}

- In phase 1, a learning styles model is analysed to create the Learning Styles Adapter Agent. The FS model was chosen as it contains descriptions of preferred teaching styles associated with each learning styles and it models the learning styles of Engineering students, such as those studying SQL. The knowledge of typical learning style preferences and associated teaching styles was extracted from the model and mapped to teaching material categories (Table 1), as described in Section 3.2. Section 4.1 will detail step 1.4 (in Table 2), the implementation of the Oscar CITS adaptation algorithm.

- In phase 2, the adaptive conversational tutorial was designed. This required the capture of knowledge from human tutors, and the modelling of a tutorial conversation. Each tutorial question was then mapped and scored according to the teaching material categories in Table 1, as described in Section 3.2. The Convagent Ltd (2005) InfoChat CA was selected as it is a text-based CA that allows information to be captured and received using variables. InfoChat adopts a pattern matching approach, which is best suited to student dialogue as it is flexible enough to cope with bad grammar, misspellings and use of 'text-chat' language. The development of CA scripts is a complex and lengthy process, and so the scripts developed for the implementation of the Oscar CITS predictive subsystem (Latham et al. 2012a) were reused and modified to provide an adaptive tutorial.

- Phase 3 entailed the construction of the Oscar CITS architecture (Fig. 1). The Oscar CITS architecture is modular, and therefore enabled the reuse of components already created for the Oscar CITS predictive subsystem (Latham et al. 2012b).

The next section will describe in more detail the final step of phase 1: the implementation of the Oscar CITS adaptation algorithm for the FS learning styles model.

\subsection{Implementing the Oscar CITS Adaptation Algorithm for the Felder-Silverman Learning Styles Model}

The generic Oscar CITS adaptation algorithm described in Section 3.3 decides the most appropriate type of adaptation to be applied for each student's individual learning path. The FS model has four learning style dimensions, each with two opposite learning styles, so there are eight learning styles plus the Neutral learning style. Learners are placed along each FS dimension according to the strength of their preference for a particular 
learning style. A study analysing the learning styles of Computing university students (Latham 2011) found the results to be consistent with the FS model, which states that on the Visual/Verbal scale most engineering students were found to be Visual learners (Felder \& Silverman 1988). Considering that Oscar CITS tutorials are conducted using a conversation (Verbal) with supporting images and diagrams throughout (Visual), and the Massa and Mayer (2006) study shows no difference in learning achievement when adapting multimedia teaching to Visual/Verbal learners, it was decided to exclude the Visual/Verbal FS dimension. Therefore for the Oscar CITS adaptation was implemented for the remaining three FS learning style dimensions: Sensor/Intuitor, Active/Reflective and Sequential/Global.

The Oscar CITS adaptation strategy states that learners with a neutral learning style class are given learning material in a mixture of styles. In the FS model, neutral learners are defined as those with an ILS questionnaire score of 1 or 3 for a learning style, indicating a low preference and placing them at the centre of the dimension.

Table 3 shows a pseudo-code representation of the adaptation algorithm applied to the three selected dimensions of the FS model. The algorithm receives a list of student learning style scores and a list of question scores and returns the best fitting adaptation for each question.

$<$ TABLE 3 HERE $>$

\section{Experimental methodology and results}

An empirical study was undertaken in a real teaching/learning environment to evaluate the success of the Oscar CITS adaptive tutoring. The experiment investigated whether the Oscar CITS adaptation algorithm was successful in improving learning by testing the hypothesis:

H: it is possible to improve learning by means of an automated online conversational tutorial by presenting tutor material adapted to a student's learning style.

To test this hypothesis it was necessary to compare the learning of a group of students who experienced a tutorial adapted to suit their learning styles with a control group. A common measure of learning is learning gain (Kelly \& Tangney 2006; Fossati, Di Eugenio, Brown, Ohlsson, Cosejo \& Chen 2009). Learning gain could be measured in a number of ways, for example the number of tutorial questions a learner answers correctly or an improvement in test scores. For the experiment, learning gain was measured in two ways:

1) By counting the number of correct answers given to tutorial questions during the tutoring conversation.

2) Using a pre-test and post-test approach (Kelly \& Tangney 2006; Fossati et al. 2009), where the same Multiple Choice Question (MCQ) test is completed before and after the tutoring conversation. Test scores are then compared to establish whether there is any improvement using:

\section{learning gain $=$ post-testScore - pre-testScore. $\quad$ (Eq. 1)}

In order to evaluate whether the Oscar CITS adaptation has a positive effect on the tutoring, it was necessary to split participants into different experimental groups. A match/mismatch approach was adopted (Tsianos et al. 2008), whereby participants are randomly assigned to follow a tutorial where the adaptation is either matched or mismatched to their learning styles. The match/mismatch approach was considered to be a better test of the adaptation than an approach where one control group experiences a basic tutorial, as it was concluded that any group experiencing additional learning material would be likely to show improved learning. The average learning gain was then compared for each experimental group to evaluate whether adapting to learning styles positively affects the success of the tutoring.

\subsection{Methodology}

Oscar CITS was integrated into a final year undergraduate computer science course at Manchester Metropolitan University. The 72 participants had previously been taught SQL but had not previously used Oscar CITS. During timetabled classes, participants were asked to review their SQL knowledge by individually working through the online revision tutorial. The revision tutorial was a formative assessment to enable students to check their understanding of SQL knowledge, to apply their knowledge to practical problems and to get instant feedback and help. The online revision tutorial was led by Oscar CITS and comprised a self-contained tutoring conversation covering ten main questions, during which all necessary learning materials, including explanations, exercises, examples, diagrams, images, movies, etc., were provided by the Oscar CITS. No additional learning materials were needed or provided by lecturers. In order to promote the completion of the tutorial, participants who completed it were awarded $2 \%$ of the module mark in recognition of engagement. Participants started the SQL revision tutorial during the class, and those who did not complete the tutorial in a single session were able to continue the tutorial via the Internet at a convenient time. This delivery style mirrors the face-to-face SQL revision session, where students work through a number of practical problems in class, with open books, and complete them at home before sitting an open-book MCQ test.

Before the start of the tutorial, each participant completed the ILS questionnaire, and depending on their learning styles was allocated to one of three experimental groups (Neutral-Adapt, Adapt and Mismatch). Participants were made aware that the tutorial would adapt depending on their responses, but were not aware 
that they would be allocated to experimental groups. Participants were assigned to the experimental groups as follows:

- Participants with no strong preference for all three FS dimensions (i.e. their ILS scores were 1 or 3) were assigned to the Neutral-Adapt group. These participants followed the neutral adaptation learning path which contains a mixture of styles.

- Participants with one or more preferred learning style were randomly assigned to either the Adapt or Mismatch group according to a 2:1 ratio. The restricted randomisation approach was adopted using a 2:1 ratio in order to achieve a balance of participant learning style characteristics as the sample of participants was small (Schultz \& Grimes 2002). These participants followed an individual adaptive learning path selected by the algorithm containing tutor material suitable for particular learning styles (e.g. containing bullet point summaries rather than textual explanations). Participants in the Mismatch group were intentionally given learning material unsuited to their learning styles by reversing their learning style scores for all FS dimensions. For example, a participant with learning style scores of Active 9 and Reflective 2 was presented with learning material adapted to the scores Active 2 and Reflective 9.

Each participant followed an individual learning path depending on their experimental group, learning styles, dialogue and existing knowledge. Throughout the tutorial, Oscar CITS recorded details of each participant's interaction (e.g. dialogue and behaviour) in log files. As well as user utterances, variables record many aspects of learner behaviour such as word count, reading time, duration and the number of interactions (full list given in Crockett et al 2011). Following the formal ILS questionnaire, participants completed a multiple choice question (MCQ) test, known as the pre-test, to assess existing SQL knowledge. During the SQL Revision conversation ten tutorial questions were presented, some incorporating multiple steps or questions, thus requiring eighteen answers. The SQL revision tutorial took on average 43 minutes. After completing the tutorial conversation, students repeated the same MCQ test, known as the post-test, and were then given overall tutor feedback and a comparison of their test results (one indication of their learning gain). Finally, students could optionally complete a user evaluation questionnaire.

On completion of the tutorials, the data gathered was analysed and the experimental group averages were compared to assess the success of the adaptation mechanism. As described previously, learning gain was measured in two ways and analysed as follows.

- Experiment 1 - Correct Tutorial Answers. This experiment considered the performance of participants during the tutorial. Eighteen answers were required for the ten tutorial questions posed. For each participant, the number of correct answers given to tutoring questions was counted, and a score out of 18 assigned. Next, the average percentage score was calculated for each experimental group and then compared to establish whether there was any difference in performance related to the adaptation applied.

- Experiment 2 - Relative MCQ Test Score Improvement. This experiment considered the average improvement in test scores as a percentage of the possible improvement, defined in (Eq. 2). This measure was selected instead of percentage learning gain (Eq. 1) as it additionally takes into account the opportunity for improvement. For example, participants who achieved 19/20 in the pre-test have less chance of improvement than those achieving 5/20, so a test score improvement of 1 mark has a different meaning if pre-test scores are ignored and such comparisons could be misleading. By relating the percentage test score improvement to the opportunity for improvement, it is possible to measure and compare how successfully different groups of participants learn from the tutorial. Average test score improvements were calculated and compared for each experimental group using the formula:

\section{learning gain / (questionCount - pre-testScore) (Eq. 2)}

\subsection{Results}

63 of the 72 participant participants fully completed the tutoring session; incomplete tutorial sessions were disregarded. Of the 63 complete tutorial sessions, one was disregarded as the participant had not engaged with the tutorial, answering 'no' to all questions and selecting the same answer for all multiple choice test questions. Table 4 shows the distribution of the 62 participants across experimental groups and the average (mean) test scores.

\section{$<$ TABLE 4 HERE $>$}

In Table 4, the ten Neutral-Adapt participants had no strong preference for any learning style (i.e. their learning styles were placed at the centre of the axis), and so followed the neutral adaptation learning path. The Adapt group contained 32 participants who followed a learning path containing a style of teaching material adapted to their preferred learning styles. The Mismatch group of 20 participants followed an adaptive learning path of teaching material that was matched to their opposite learning styles. The Mismatch group had a slightly lower average pre-test score (out of 12), but the average post-test scores were approximately the same for all participants across the sample. 
As seen in Table 4, the Oscar CITS tutorial had a positive impact on test scores across all groups, regardless of the type of adaptation employed. The average learning gain (calculated using (Eq. 1)) over all participants was $19 \%$.

Table 5 reports the results of the experiments, which were tested for difference between the experimental groups using the Kruskal-Wallis test with a confidence interval of 95\%. The Kruskal-Wallis test is nonparametric so it does not require normality and works with data represented as percentages (unlike the more common ANOVA).

$<$ TABLE 5 HERE $>$

\subsubsection{Experiment 1 - Correct Tutorial Answers}

Participants in the Neutral-Adapt and Adapt groups have averages of $73 \%$ and $71 \%$ correct answers given during the tutoring. Conversely, the Mismatch group average of only $61 \%$ correct answers is much lower at $12 \%$ less than the Adapt group. The test results gave a Kruskal-Wallis statistic of 10.31 and a p-value of 0.006 , indicating a significant difference for the Mismatch group.

The results suggest that participants who are presented with learning material matched to their individual learning styles perform significantly better (on average 12\%) than participants using learning material that is not matched to their learning styles. The results therefore support the hypothesis $\mathrm{H}$ as the effectiveness of the tutoring has been improved by adapting to a participant's learning style.

\subsubsection{Experiment 2 - Relative MCQ Test Score Improvement}

When comparing the relative test score improvement, participants in the Adapt group improved on average $3 \%$ more than those in the Mismatch group. This result supports the hypothesis that adapting to learning styles improves the effectiveness of tutoring. However, the Kruskal-Wallis statistic of 0.33 and p-value of 0.849 show that the difference is not significant.

Participants in the Neutral-Adapt group improved the least, at $61 \%$, with a sample average of $63 \%$ improvement in test scores when considering the opportunity for improvement. Again, the results show that the Oscar tutorial has had a positive effect on participant test scores.

\subsection{Discussion}

On the whole, the results have shown that the Oscar CITS successfully tutors learners, with an average learning gain of $19 \%$ across all participants. The results of the participant evaluation questionnaire revealed that use of the Oscar CITS was welcomed, with $94 \%$ of learners finding the tutoring helpful and $90 \%$ agreeing that Oscar helped them to revise. $92 \%$ of participants would use Oscar CITS if it were available, with $78 \%$ saying they would prefer Oscar over learning from a book. Unexpectedly, 46\% of the sample said they would use Oscar rather than attending face-to-face tutorials. There were no significant differences in feedback between the experimental groups.

To evaluate whether the Oscar CITS adaptation algorithm was successful in improving learning, two different measures were used to compare learning between experimental groups. Learning gain was measured during the learning process and using a summative assessment. In Experiment 1, learning was measured within the tutorial itself by counting the number of correct answers participants gave to tutorial questions. This measure evaluated how well participants received and understood the learning material at the time it was presented, in order to establish whether matching the style of teaching material to preferred learning style improved learning. The analysis of results using this measure showed a significant difference in the achievements of participants in the Adapt group compared to those in the Mismatch group, with the Adapt group performing on average 12\% better. In Experiment 2, learning was measured in an assessment situation at the end of the tutorial by considering the relative improvement in test results. The measure factored in each participant's prior knowledge by relating test score improvements to the opportunity for improvement, i.e. their pre-test score (as described in Section 5.1). The results for Experiment 2 were inconclusive, showing a small but not significant difference, with the Adapt group performing on average 3\% better than the Mismatch group. This insignificant result could reflect the fact that learning was measured at the end of the tutorial rather than at the time the adaptive learning material was presented. As part of the Oscar CITS tutorial, learners are given intelligent support and feedback on their responses. If learners are unable to answer correctly after several attempts, Oscar CITS explains the correct answer. This instant feedback on erroneous and incomplete answers is designed to improve learning, so even where a participant is unable to give the correct answer during the tutorial, the intelligent feedback they are given on wrong answers would be expected to improve their learning and test scores. It may be possible to explore this measure further by removing the feedback mechanism for incorrect answers during the tutorial, however as experiments were conducted with real students in a real teaching/learning environment this would also remove some of the benefits of using Oscar CITS.

In summary, the adaptation to learning styles was successful as experiment 1 showed a statistically significant 
difference between the learning gain in the Adapt and Mismatch groups. The hypothesis has been supported by the results of Experiment 1, however the results of Experiment 2 are inconclusive. The results show that adapting to participant learning styles during an automated conversational tutorial has improved learning gain by $12 \%$ (Experiment 1$)$ and $3 \%$ (Experiment 2 ).

A comparison with other CITS is not possible, as no other CITS can adapt their tutoring style to match an individual's learning styles (as stated earlier). There are several ITS without natural language interfaces that adapt to learning styles, however evaluation of the effect of adaptation differs. For example, Sangineto et al. (2007) and Carver et al. (1999) adapt according to the Felder-Silverman model, but use qualitative feedback from a questionnaire to compare differences in non-adaptive and adaptive user experiences. In EDUCE (Kelly \& Tangney 2006), adaptation is to the Multiple Intelligence model, and although learning gain was investigated, the results showed a higher learning gain for learners with mismatched learning material. The Adaptive Web System (Tsianos et al. 2008) adapts to cognitive style and emotion, and was found to improve learning performance.

\section{Generalising the Oscar CITS Adaptation Algorithm}

The research aim was to develop a generic approach to adaptation that could be applied to other learning styles models and subject domains. In developing the Oscar CITS adaptation algorithm, knowledge was extracted from an example learning styles model and analysed. This analysis of learning styles knowledge enabled the development of several teaching material categories that relate to learner behaviours rather than the subject domain and learning styles model. By extracting knowledge from a different learning styles model (such as Honey and Mumford 1992), the teaching material categories could be mapped to different learning styles. For example, in the Honey and Mumford model, Pragmatists like to try out and practice techniques so that learning style could be linked to the teaching material category 5, Practical exercises. A further study is required to test the applicability of this adaptation approach to a different domain and learning styles model.

The independent teaching material categories were used to design a strategy for scoring learning materials that indicates the availability and strength of adaptation available for each learning style in the chosen model. The Oscar CITS adaptation algorithm then combines the strength of an individual's learning style preference with the availability of adaptive teaching material to decide the best fitting adaptation for an individual for each tutoring question.

The main limitation of the Oscar CITS adaptation approach is the time and expertise needed for the knowledge engineering of the learning styles model (described in Section 3.2). In this case study the task was less onerous as the FS model clearly matches teaching styles descriptions to the learning styles in the model; this knowledge may not be included in other learning styles models. Although it is hoped that the teaching material categories developed by expert lecturers can be applied to other learning styles models, this step may still be lengthy. Time and workload are a limitation of all adaptive ITS, as it is necessary to produce multiple copies of learning material in different styles. In CITS this problem is compounded by the additional need for CA experts to produce and test conversation scripts covering multiple styles of natural language tutoring. Further work is needed to reduce the burden of scripting, either through the production of tools or of more efficient scripting mechanisms such as the use of semantic conversational agents (O’Shea, Bandar \& Crockett 2009).

Despite being developed for natural language tutorials such as the Oscar CITS, the adaptation algorithm could be applied to ITS or other adaptive systems as it selects the best fitting solution based on two independently scored factors. As the Oscar CITS adaptation algorithm is generic, it could be applied to any learning styles model where it is possible to score the strength of the individual's preference. Furthermore, the algorithm could be used to adapt to other individual factors that can be scored. For example boredom and frustration are emotions that affect learning and can be scored to represent the strength of feeling, thus the algorithm could be used to adapt to these emotional factors. Further work is required to investigate the success of the algorithm in selecting adaptations for other types of e-learning system and other individual factors.

\section{Conclusions}

Oscar CITS is an innovative conversational ITS that directs a tutorial conversation and dynamically predicts and adapts its tutoring to an individual's learning styles. Oscar CITS is currently being used by students at Manchester Metropolitan University to support learning. This paper has described the development of the novel Oscar CITS adaptation technique which determines the most appropriate adaptation to apply for an individual's learning path, selecting the best adaptation for each tutorial question independently. This approach results in a varied learning experience suited to both the individual and the style of question, thus helping to promote engagement. The Oscar CITS adaptation algorithm uniquely combines several aspects of the learner's preferences (e.g. learning styles) with the availability of adaptive learning material to find the best fitting adaptation. The incorporation of the availability of adaptive learning material in the algorithm enables adaptive tutorials to be produced incrementally, thus addressing a considerable development barrier. The Oscar CITS adaptation algorithm is independent of the adopted learning styles model (or other social factor, e.g. emotion), 
and further work is planned to test its applicability to other ITS and learning styles models.

A case study was described where Oscar CITS was implemented for the SQL domain and the FelderSilverman learning styles model. The Oscar CITS adaptation algorithm was applied to the FS learning styles model. An adaptive conversational tutorial to revise SQL was created and the Oscar CITS adaptation algorithm was empirically tested by students in a real life learning environment. During the experiment, 72 students were unknowingly assigned to experimental groups according to the adaptation to be applied - the Neutral-Adapt and Adapt groups experienced a tutorial suited to their learning styles, whereas the Mismatch group undertook a tutorial that did not suit their learning styles. Each group's tutorials were compared in two experiments designed to test whether adapting to learning styles improved the learning experience. The Oscar CITS tutorial was positively received by students, who improved their test scores by an average of $19 \%$.

The results of experiment 1 have shown that there is a marked difference in the achievements of students during the tutorial. In experiment 1 the Adapt group performed significantly better (on average 12\%) than the Mismatch group during the tutorial, and in experiment 2 the Adapt group improved test scores on average 3\% better than the Mismatch group. These results indicate that it is possible to improve learning by adapting to an individual's learning styles during tutoring.

It is concluded that the adaptation algorithm developed for the Oscar CITS has made a positive difference in student learning experiences. Students whose tutorial was adapted to match their learning styles performed on average $12 \%$ better during the tutorial and improved test scores by an average of 3\% more than students who were presented with a tutorial not suited to their learning styles. The $12 \%$ better performance by the Adapt group in experiment 1 was shown to be a statistically significant difference. On the whole, the Oscar CITS tutorial produced positive results, with test scores across the sample an average $19 \%$ better following the tutorial.

\section{Appendix A}

\section{$<$ TABLE A HERE $>$}

Following the Oscar CITS adaptation algorithm, if the learner is classed as Neutral for all learning style dimensions, they follow the Neutral learning path for all tutorial questions. Otherwise, for each tutorial question:

- For each learning style class $\boldsymbol{c}$ a learner question score $\boldsymbol{a}_{c}$ is defined as the product of the question score for that learning style $\boldsymbol{q}_{\boldsymbol{c}}$ and the learner learning style score $\boldsymbol{s}_{\boldsymbol{c}}$.

- The learning style class $\boldsymbol{c}$ with the highest learner question score $\boldsymbol{a}_{c}$ wins.

Additional rules are followed for conflict resolution, i.e. determining the adaptation when more than one maximum score $\boldsymbol{a}$ exists in $\boldsymbol{W}$, the set of winning scores:

- In the case where there is no clear winner (i.e. no single maximum score $\boldsymbol{a}$ ), the learning style class in $\boldsymbol{W}$ with the maximum learner score $\boldsymbol{s}$ wins (i.e. the learner's strongest learning style preference in the set of winners).

- If there is no clear winner again (i.e. the learner has more than one learning style with the maximum score $\boldsymbol{s})$, the question scores $\boldsymbol{q}$ for the learning style classes in $\boldsymbol{W}$ are compared, and the maximum question score $\mathbf{q}$ wins.

- Finally, if there is still no winner, a learning style adaptation is selected randomly from the learning style classes in $\boldsymbol{W}$.

\section{Appendix B}

\section{$<$ TABLE B HERE $>$}

This appendix shows examples demonstrating the adaptations selected by the algorithm (applied to the FS model) and of conflict resolution. In the table, first the question learning style scores (q) are shown for three tutorial questions. Next, each learner's learning style dimension class and score $\langle\boldsymbol{c}, \boldsymbol{s}\rangle$ is listed, followed by (for each tutorial question) the calculated learner question scores $(\boldsymbol{a})$, the set of winners $(\boldsymbol{W})$ and the resulting adaptation. The strength of learning style preference is scored by the ILS as 1, 3, 5, 7, 9 or 11 . Neutral learners have low preference for a learning style, i.e. ILS scores of 1 or 3.

Each example in the table will now be described:

- Learner 1 has no strong preference for any of the FS dimensions, scoring 1 or 3 , and so is classified as Neutral for all dimensions. Following the algorithm the neutral adaptation is applied to all tutorial questions.

- Learner 2 has strong preferences for all FS dimensions. For Q1, the learner learning style score (shown first) is multiplied by the question learning style score, giving a learner question score for that FS dimension. The winner is the FS dimension with the highest score; for Q1 the Active adaptation. For Q2 the winning adaptation is Sequential because, even though the learner has a stronger preference for the Active learning style, the tutorial question offers more adaptations for the Sequential learning style. For Q3 there 
are two winners (Visual and Sequential), so first the learner learning style scores are compared, followed by the tutorial question scores. As all scores are equal, the adaptation is selected randomly from the two winners, resulting in a Visual adaptation.

- Learner 3 has a low preference for two FS dimensions (Intuitive and Visual) and a moderate preference for the Active and Global learning styles. For Q1, there are two winners, and as the learner and question scores are equal, the Active adaptation was selected at random. For Q2, there are three winners, so the Global adaptation wins as it has the highest learner learning style score. Q3 only adapts to three learning styles, so the clear winner is the Visual adaptation in this case.

\section{References}

Akbulut, Y. \& Cardak, C.S. (2012). Adaptive educational hypermedia accommodating learning styles: A content analysis of publications from 2000 to 2011. Computers \& Education 58, 835-842.

Brusilovsky, P. \& Peylo, C. (2003). Adaptive and Intelligent Web-based Educational Systems. Int. J. Artificial Intelligence in Education 13, 156-169.

Carver, C.A. Howard, R.A. \& Lane, W.D. (1999). Enhancing Student Learning Through Hypermedia Courseware and Incorporation of Learning Styles. IEEE Trans. Education 42 (1), 33-38.

Chi, M.T.H., Siler, S., Jeong, H., Yamauchi, T. \& Hausmann, R.G. (2001). Learning from human tutoring. Cognitive Science 25, $471-533$. Coffield F., Moseley D., Hall E. \& Ecclestone K. (2004). Learning Styles and Pedagogy in Post-16 Learning: A Systematic and Critical Review. London: Learning and Skills Research Center.

Convagent Ltd (2005), Convagent, Available: http://www.convagent.com/ [10 Jan. 2012].

Craig, S.D., Hu, X., Graesser, A.C., Bargagliotti, A.E., Sterbinsky, A., Cheney, K.R. \& Okwumabua, T. (2013). The impact of a technologybased mathematics after-school program using ALEKS on student's knowledge and behaviours. Computers \& Education 68, $495-504$. Crockett, K., Latham, A., McLean, D., Bandar, Z. \& O’Shea, J. (2011). On Predicting Learning Styles in a Conversational Intelligent Tutoring System using Fuzzy Classification Trees. In: Proc. IEEE Int'l Conf. Fuzzy Systems (WCCI '11), 2481-2488.

Dzikovska, M.O., Bental, D., Moore, J.D., Steinhauser, N.B., Campbell, G.E., Farrow, E. \& Callaway, C.B. (2010). Intelligent tutoring with Natural Language Support in Beetle II System. LNCS 6383, 620-625

Felder, R. M. \& Brent, R. (2005). Understanding Student Differences. J. Engineering Education 94 (1), 57-72.

Felder, R. \& Silverman, L.K. (1988). Learning and Teaching Styles in Engineering Education. J. Engineering Education 78 (7), $674-681$. Felder, R.M. \& Soloman, B.A. (2008). Index of Learning Styles questionnaire. Available: www.ncsu.edu/felder-public/ILSpage.html, [26 Apr. 2008].

Fossati, D., Di Eugenio, B., Brown, C.W., Ohlsson, S., Cosejo, D.G. \& Chen, L. (2009). Supporting Computer Science Curriculum: Exploring and Learning Linked Lists with iList. IEEE Trans. Learning Technologies 2 (2), 107-120.

Garcia, P., Amandi, A., Schiaffino, S. \& Campo, M. (2007). Evaluating Bayesian networks' precision for detecting students' learning styles. Computers \& Education 49, 794-808.

Graesser, A., Chipman, P., Haynes, B.C. \& Olney, A. (2005). AutoTutor: An Intelligent Tutoring System With Mixed-Initiative Dialogue. IEEE Trans. Education 48 (4), 612-618.

Honey, P. \& Mumford, A. (1992). The manual of learning styles, Peter Honey, Maidenhead.

Jones, A. \& Issroff, K. (2005). Learning technologies: Affective and social issues in computer-supported collaborative learning. Computers \& Education 44, 395-408.

Kelly, D. \& Tangney, B. (2006). Adapting to intelligence profile in an adaptive educational system. Interacting with Computers 18, 385409.

Klasnja-Milicevic, A., Vesin, B., Ivanovic, M. \& Budimac, Z. (2011). E-Learning personalization based on hybrid recommendation strategy and learning style identification. Computers \& Education 56, 885-899.

Latham, A. (2011). Personalising Learning with Dynamic Prediction and Adaptation to Learning Styles in a Conversational Intelligent Tutoring System. PhD thesis, School of Computing, Mathematics and Digital Technology, Manchester Metropolitan Univ., Manchester, UK. Latham, A.M., Crockett, K.A., McLean, D.A. and Edmonds, B. (2012a). A Conversational Intelligent Tutoring System to Automatically Predict Learning Styles. Computers \& Education 59 (1), 95-109.

Latham, A.M., Crockett, K.A., McLean, D.A. and Edmonds, B. (2012b). Adaptive Tutoring in an Intelligent Conversational Agent System. In N.T. Nguyen, Transactions on Computational Collective Intelligence VIII, LNCS 7430 (pp. 148-167). Berlin: Springer.

Lee, L.H., Isa, D., Choo, W.O. \& Chue, W.Y. (2012). High relevance keyword extraction facility for bayesian text classification on different domains of varying characteristic. Expert System with Applications 39, 1147-1155.

Lehman, B., D’Mello, S. K., \& Graesser, A. C. (2012). Confusion and Complex Learning during Interactions with Computer Learning Environments. The Internet and Higher Education 15(3), 184-194.

Leontidis, M., Halatsis, C., Grigoriadou, M. (2011). Using an affective multimedia learning framework for distance learning to motivate the learner effectively. Int. J. Learning Technology 6 (3), 223-250.

Li, Y., McLean, D., Bandar, Z., O’Shea, J. \& Crockett, K. (2006). Sentence similarity using semantic nets and corpus statistics. IEEE Trans. Knowledge and Data Eng. 18 (8), 1138-1150.

Mao, X. \& Li, Z. (2010). Agent based affective tutoring systems: A pilot study. Computers \& Education 55, $202-208$.

Massa, L.J. \& Mayer, R.E.(2006). Testing the ATI hypothesis: Should multimedia instruction accommodate verbalizer-visualizer cognitive style?. Learning and Individual Differences 16, 321-336.

Moreno, R., Mayer, R.E., Spires, H. \& Lester, J. (2001). The case for social agency in computer-based teaching: Do students learn more deeply when they interact with pedagogical agents? Cognition and Instruction 19, 177-213.

O’Shea, J., Bandar, Z. \& Crockett, K. (2011), Systems Engineering and Conversational Agents, in Tolk, A. \& Jain, L.C. (Eds), IntelligenceBased Systems Engineering, Intelligent Systems Reference Library 10, Springer-Verlag Berlin Heidelberg.

O’Shea, K., Bandar, Z. \& Crockett, K. (2009), Towards a New Generation of Conversational Agents Based on Sentence Similarity. Advances in Electrical Engineering and Computational Science 39, 505-514.

Pahl, C. \& Kenny, C. (2009). Interactive Correction and Recommendation for Computer Language Learning and Training. IEEE Trans. Knowledge and Data Eng. 21 (6), 854-866.

Popescu, E. (2010). Adaptation provisioning with respect to learning styles in a Web-based educational system: an experimental study. Journal of Computer Assisted Learning 26, 243-257.

Rahati, A. \& Kabanza, F. (2010). Persuasive Dialogues in an Intelligent Tutoring System for Medical Diagnosis. LNCS 6095, 51-61. 
Redondo, M.A., Bravo, C., Ortega, M. \& Verdejo, M.F. (2007). Providing adaptation and guidance for design learning by problem solving: The design planning approach in DomoSim-TPC environment. Computers \& Education 48, 642-657.

Sammut, C. (2001). Managing Context in a Conversational Agent. Linkoping Electronic Articles in Computer \& Information Science 3 (7). Linkoping University Electronic Press, Sweden.

Sanders, D.A. \& Bergasa-Suso, J. (2010). Inferring Learning Style from the way Students Interact with a Computer User Interface and the WWW. IEEE Trans. Education 53 (4), 613-620.

Sangineto, E., Capuano, N., Gaeta, M. \& Micarelli, A.(2007). Adaptive course generation through learning styles representation. $J$.

Universal Access in the Information Society 7 (1), 1-23.

Schultz, K.F. \& Grimes, D.A. (2002). Generation of allocation sequences in randomised trials: chance, not choice. The Lancet 359, 515-519.

Stankov, S., Rosić, M., Žitko, B. \& Grubišić, A, (2008). TEx-Sys model for building intelligent tutoring systems. Computers \& Education 51, 1017-1036.

Triantafillou, E., Pomportsis, A., Demetriadis, S. \& Georgiadou, E. (2004). The value of adaptivity based on cognitive style: an empirical study. British J. Educational Technology 35 (1), 95-106.

Tsianos, N., Lekkas, Z., Germanakos, P., Mourlas, C. \& Samaras, G. (2008). User-centered Profiling on the basis of Cognitive and

Emotional Characteristics: An Empirical Study. LNCS 5149, 214-223.

Wallace, R.S. (2009). The Anatomy of A.L.I.C.E. In R. Epstein, G. Roberts \& G. Beber, Parsing the Turing Test Part III (pp. 181-210).

Berlin: Springer.

Wang, S. \& Wu, P. (2008). The role of feedback and self-efficacy on web-based learning: The social cognitive perspective. Computers \& Education 51, 1589-1598. 